\title{
CÓMO LOS ESTUDIANTES DE LA FORMACIÓN INICIAL DEL PROFESORADO PERCIBEN Y TRATAN LA EVALUACIÓN FORMATIVA Y COMPARTIDA
}

How students from initial teacher education understand and deal with formative and shared assessment

Como os estudantes da formação inicial de professores percebem e tratam as avaliações formativa e partilhada

André Pisco de Moura (1)

Paula Fazendeiro Batista (2)

Víctor M. López-Pastor (3)

Financial support: The work of the first author is supported by Fundação para a Ciência e Tecnologia (FCT). PhD grant: SFRH/BD/137848/2018

(1) Universidad de Porto, Facultad de Deporte, Portugal. Teléfono: +351 917522046. Correo electrónico: luisandrepmoura@hotmail.com

(2) Universidad de Porto, Facultad de Deporte, Portugal. Centro de Investigación de Educación, Innovación e Intervención en lo Deporte (CIFI2D) Teléfono: +351 966342447. Email: paulabatista@fade.up.pt

(3) Universidad de Valladolid, España. Teléfono: +34 921112321. Correo electrónico: vlopez@mpc.uva.es

\section{Resumen}

El punto de partida de este estudio fue la estancia de dos meses de un estudiante portugués de doctorado interesado en profundizar su conocimiento sobre los procesos de evaluación en una facultad en España. Este trabajo pretende captar los entendimientos y las valoraciones que los estudiantes otorgan a las evaluaciones formativas y compartidas (EFyC) realizadas por el tercer autor $\left(1^{\circ}\right.$ y $3^{\circ}$ curso, Grado de Maestro en Educación Infantil). Ocho estudiantes con diferentes niveles de participación, interés e implicación fueron seleccionados. Las entrevistas semiestructuradas individuales fueron analizadas mediante análisis temático. Los estudiantes entienden adecuadamente los procesos desarrollados en esta asignatura, aunque tengan diferentes interpretaciones sobre los mismos. Ellos identifican ventajas en los procesos de EFyC, tanto como alumnos como en su futuro como maestros. Apuntan algunas dificultades para emplearlos y una gran carga de trabajo. Los 
estudiantes, han dicho que si estuviesen como maestros en la escuela aplicarían estas evaluaciones.

Palabras clave: Investigador externo; procesos de evaluación formativa y compartida; formación del profesorado; percepciones de futuros maestros

\begin{abstract}
The starting point of this this study was the coming of a Portuguese $\mathrm{PhD}$ student, for two months to a faculty in Spain, with interest in deepening its knowledge about assessment processes. This study aims to capture the understandings and valuation given by the students to the formative and shared assessment teaches by the third author $\left(1^{\text {st }}\right.$ and $3^{\text {rd }}$ years of Bachelor to teach in Kindergarten Education). Eight students were selected according their participation level, interest and commitment. Data were collected individually through semi-structured interviews and analyzed through thematic analysis. Students revealed a correct understanding of the processes developed on this subject; despite they have different perceptions about it. They identified advantages on the use of formative and shared assessment processes. They also reported some problems to implement these processes and a heavy workload. Students referred that if they were at school as teachers, they would implement both assessments.

Keywords: External researcher; formative and shared assessment process; teacher education; future teachers' perceptions
\end{abstract}

\title{
Resumo
}

Partindo do interesse investigativo de um estudante português de doutoramento na temática da avaliação que veio durante dois meses a uma faculdade em Espanha, este estudo teve como objetivo captar os entendimentos e valoração atribuída pelos estudantes à disciplina lecionada pelo terceiro autor do estudo $\left(1 .^{\circ}\right.$ e $3 .^{\circ}$ ano da licenciatura em Educação Pré-Escolar), a respeito do sistema de avaliação formativa e partilhada utilizado na mesma. Oito estudantes foram selecionados pelo investigador, tendo em conta, o seu nível de participação, interesse e empenho nas aulas. As entrevistas semiestruturadas individuais foram analisadas através de análise temática. Os estudantes evidenciaram um entendimento adequado dos processos desenvolvidos nesta disciplina, embora tenham diferentes interpretações sobre o mesmo. Identificaram vantagens nos processos de avaliação formativa e partilhada, quer como alunos, quer 
como futuros professores. Apontam algumas dificuldades para implementar estes processos e um grande volume de trabalho. Os estudantes disseram que se estivessem na escola como professores aplicariam estas modalidades de avaliação.

Palavras-chave: Investigador externo; processos de avaliação formativa e partilhada; formação de professores; perceções de futuros professores

\section{Introducción y contextualización}

Varios autores tienen identificada la importancia de la evaluación para las diversas dimensiones del proceso enseñanza-aprendizaje (Baird, Andrich, Hopfenbeck \& Stobart, 2017; Penney, Brooker, Hay \& Gillespie, 2009).

En España se están desarrollando experiencias de evaluación formativa y compartida $($ EFyC) en educación superior, que apuntan hacia una mejoría del aprendizaje (Hamodi, López-Pastor \& López-Pastor, 2017; López-Pastor, 2011; Pueyo et al., 2008). Teniendo en cuenta el interés indagatorio de un estudiante de doctorado portugués, en la temática de la evaluación para el aprendizaje en la formación inicial del profesorado, este trabajo tuvo como propósito evaluar cómo los estudiantes de la formación inicial del profesorado entienden y valoran el sistema de EFyC utilizado en la asignatura del tercer autor de este estudio.

\section{Diseño y desarrollo}

Este trabajo se desarrolló durante los dos meses de estancia de investigación de este estudiante en la Facultad de Educación de Segovia (España).

Se llevaron a cabo entrevistas semiestructuradas individuales a ocho estudiantes de una asignatura del $1^{\circ}$ y $3^{\circ}$ curso del Grado de Maestro en Educación Infantil. Los datos de las entrevistas fueron tratados a través de análisis temático. Los estudiantes fueron seleccionados por el investigador de acuerdo con su nivel de participación, interés e implicación en las clases.

\section{Resultados}

Los estudiantes parecen estar de acuerdo en que la Evaluación Formativa (EF) los alumnos participan de manera pasiva en la evaluación y que su misión es escuchar el feedback del profesor para mejorar, mientras que el profesor es el responsable por corregir y señalar que está mal:

Como los estudiantes de la formación inicial del profesorado perciben y tratan la evaluación formativa y 
Estudiante7- "El alumno no tiene participación. Es algo sólo del profesor".

Así consideran que la EF se desarrolla durante el proceso enseñanza-aprendizaje y tiene como objetivo identificar las dificultades de los alumnos y corregir sus fallos para mejorar su aprendizaje y, por tanto, no tiene como finalidad principal calificar:

Estudiante2- "Es una evaluación que te hacen a lo largo del curso para decirte como vas y poder mejorar algunos aspectos. El objetivo es mejorar lo que estás haciendo mal y seguir haciendo bien lo que ya sabes".

Uno de los estudiantes considera que EF incluye autoevaluación, evaluación profesoralumno y alumno-alumno, por lo que los alumnos son participantes activos del proceso:

Estudiante5- "El alumno aprende a partir de sus errores, a partir de la evaluación. Tanto la evaluación del profesor al alumno, como la evaluación entre el alumnado o corregirse el mismo su aprendizaje".

Sin embargo, la mayoría refiere que estas formas de evaluación son parte de la evaluación compartida, donde el proceso es bidireccional, dialogado entre profesor y alumno. Dos estudiantes consideran la autoevaluación como una evaluación formativa. A pesar de esas diferencias, apuntan que las dos tienen el objetivo común de querer que los alumnos aprendan y que se puedan desarrollar durante el proceso de aprendizaje:

Estudiante6- "Se parecen en que las dos evaluaciones sirven para mejorar y ver en que se falla para no volver a cometerlo; y se diferencian, en que la formativa únicamente tiene una dirección".

Sobre lo que estas evaluaciones aportan, los estudiantes tienen diferentes perspectivas; unos creen que la EF tiene más contribuciones a nivel personal, social o cognitivo, mientras que otros ven esas contribuciones cuándo es empleada la Evaluación Compartida. Otra perspectiva es que la EF está más orientada hacia los objetivos, mientras que la compartida desarrolla competencias docentes:

Estudiante4- "Formativa está más enfocada en los objetivos que se tienen que aprender".

Estudiante7- "Ver la percepción que tiene el alumnado de su propio aprendizaje, que cree que ha adquirido y de qué forma".

Sobre el trabajo que desarrollan en esta facultad, los estudiantes identifican varias ventajas en su aprendizaje como estudiantes y como futuros maestros. Según ellos, tienen la posibilidad de ir mejorando su trabajo y están más motivados e implicados, porque son participantes activos del proceso. Reconocen que el proceso es más 
transparente y hay un mayor aprendizaje, porque los conocimientos son puestos en práctica y reflexionan sobre ellos, lo que te prepara mejor para el futuro como maestro.

Estudiante1- "Te das más cuenta donde son los fallos. Donde tienes que mejorar. Te paras más a pensar las cosas. Motivación más alta, así como la implicación. Más reflexión”.

Estudiante8- "Lo más importante en estas evaluaciones es que pones en práctica los conocimientos teóricos. Si tienes conocimientos teóricos y no los usas, no los adquieres en su totalidad y en el momento que tienes que empléalos, no estás preparado".

Como principales desventajas, apuntan que algunas clases prácticas las imparten sus compañeros en vez de los profesores y que la contribución entre iguales depende de quién sea el compañero:

Estudiante8- "La desventaja es que la clase no te está dando un profesional. Tú como alumno estás recibiendo una clase de aprendices y no de un profesional".

Estudiante2- “¿Puedes aprender más con la evaluación? Puede que sí o puede que no. Siempre depende del compañero que te corrige".

Como principales ventajas refieren que los profesores les dan un guion de lo que tienen que hacer y les facilitan material. Como es un proceso abierto (pueden hacer cambios en los documentos que entregan) y compartido (entre grupos de trabajo y profesoresalumnos) ellos aprenden más:

Estudiante8- "Facilidades son los textos que te están dando que directamente te aporta una fuente de información que se supone que es de otro experto".

Estudiante3- "Los profesores permiten que cambiemos lo que entregamos, te dicen lo que está mal y tienes que corregir. Eso facilita más el aprendizaje del alumno que darle sólo una nota".

Estos estudiantes indican que si ejerciesen como maestros en la escuela aplicarían estas dos evaluaciones, porque permiten mejorar su trabajo, centrarlo más en el alumno, acompañar su aprendizaje y que estos sean conscientes del mismo.

Estudiante7- "Si fuera profesora haría las dos, para que los niños también puedan expresar sus opiniones acerca de lo que han aprendido y las dificultades que han tenido. Seguramente mañana, tengo más alumnos y puedo ir mejorando mi trabajo".

\section{Conclusiones}

En general los estudiantes parecen entender adecuadamente los sistemas de evaluación desarrollados en esta asignatura, aunque haya diferentes perspectivas sobre los mismos. 
El alumnado considera que la EFyC reporta grandes ventajas, tanto como alumnos como en su futuro como maestros. Identifican también algunos problemas cuándo tienen que emplearlos y consideran que exige una gran carga de trabajo. Comentan que si trabajaran como maestros en la escuela utilizarían estas evaluaciones.

\section{Referencias}

Baird, J.-A., Andrich, D., Hopfenbeck, T. N., \& Stobart, G. (2017). Assessment and learning: fields apart? Assessment in Education: Principles, Policy \& Practice, 24(3), 317-350. doi:10.1080/0969594X.2017.1319337

Hamodi, C., López-Pastor, V. M., \& López-Pastor, A. T. (2017). If I experience formative assessment whilst studying at university, will I put it into practice later as a teacher? Formative and shared assessment in Initial Teacher Education (ITE). European Journal of Teacher Education, 40(2): 171-190.

López-Pastor, V. M. (2011). El papel de la evaluación formativa en la evaluación por competencias: aportaciones de la red de evaluación formativa y compartida en docencia universitaria. Revista de Docencia Universitaria, 9(1), 159-173.

Penney, D., Brooker, R., Hay, P., \& Gillespie, L. (2009). Curriculum, pedagogy and assessment: three message systems of schooling and dimensions of quality physical education. Sport, Education and Society, 14(4), 421-442. doi:10.1080/13573320903217125

Pueyo, Á. P., Sánchez, B. T., López-Pastor, V. M., Ortín, N. U., Lara, E. R., Bujosa, M. C., Fernández, N. G., \& Castejón, F. J. (2008). Formative and shared assessment in higher education and European area of higher education: Key questions for their implementation. Revista de Educación, 347, 435-451. 